\section{Indemnity cover additional benefit}

Indemnity is no one's favourite topic, not least as it can be confusing and complex, but the British Society of Dental Hygiene and Therapy (BSDHT) says it is endeavouring to make getting the right protection easier for all its full members.

Designed specifically for dental hygienists and dental therapists by the BSDHT and Howden chartered insurance broker, full membership of the BSDHT offers the additional benefit of indemnity cover - a policy that ensures value without compromising on quality.

It covers a range of areas as standard, including:

- Professional indemnity

- Full scope of practice

- Breach of professional confidentiality

- Direct access

- Helpline for advice and support

- GDC Investigation costs

- Automatic run-off for 12 months for death, retirement and disability

- Automatic run-off for 12 months for maternity or paternity leave, where insurance is not renewed

- Teeth whitening under prescription.

It is also possible to add on Public Liability cover, as well as botulinum toxin and dermal filler services.

More information about the BSDHT is available at www.bsdht.uk or by calling 01788575050 or emailing enquiries@bsdht.org.uk.

\section{'Royal' restorations}

Supreme restorations that are said to be fit for a king are available with the fully integrated CAD/CAM system of MyCrown.

Boasting an acquisition unit of both $2 \mathrm{D}$ and $3 \mathrm{D}$ capabilities, as well as a state-of-the-art milling unit, MyCrown provides the facility for practitioners to create everyday restorations including crowns, veneers, inlays, onlays and small bridges.

Complete with touchscreen technology, advanced software and a fully guided workflow, the manufacturer says that producing beautiful restorations has never been simpler.

Using MyCrown's sophisticated intraoral scanner, practitioners follow a simple five-step guided process to guarantee efficient planning and smooth delivery of restorative solutions which can be fine-tuned to ensure the ideal fit for each patient.

\section{How to handle halitosis}

Halitosis is a sensitive subject, but can be handled gently and effectively by recommending CB12 White mouthwash as part of oral health instruction.

Volatile sulphur compounds (VSCs) are mainly responsible for halitosis and to keep these as well as plaque and bacteria under control, they need a clinically proven mouthwash.

CB12 White contains all necessary ingredients to target and neutralise odour-causing VSCs with long lasting effects. It also contains anti-plaque agents and fluoride to strengthen teeth and prevent decay.

The added bonus with CB12 White is that it also has the technology to lift

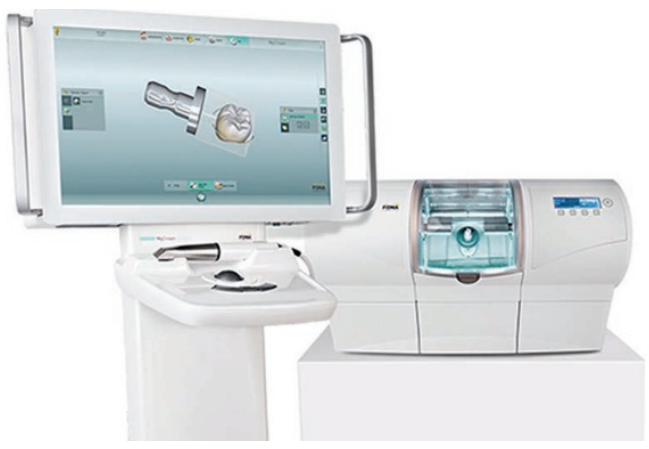

MyCrown eliminates the need to use traditional impression methods and offers practitioners the freedom to choose restoration blocks for specific requirements without limiting them to a single manufacturer or material.

The system also allows the user to save detailed designs for future reference.

More information is available at www. my-crown.co.uk or by calling 03339873007 .

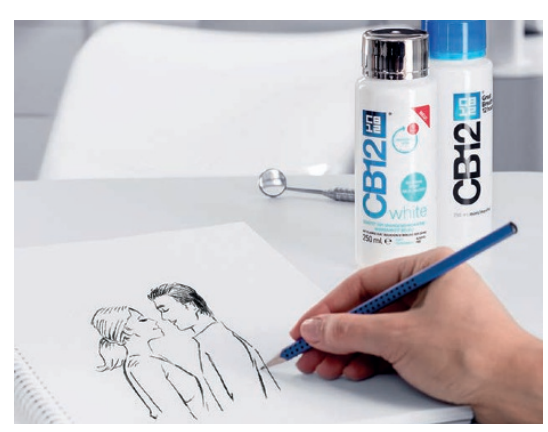

tooth stains and prevent new stains from developing.

Patients who use CB12 White twice a day for two weeks will notice a pleasing whitening effect as well as pleasant breath.

More information about $\mathrm{CB} 12$ is available at www.cb12.com.

\title{
New ergonomic tray design
}

Ancar has produced its new auxiliary tray to support weights of up to $4 \mathrm{~kg}$ in what is an exclusive design that makes it stand out among other high-end products.

Ancar's latest innovation is its new model of auxiliary tray with a useful surface area of $290 \times 375 \mathrm{~mm}$. This accessory can support from 0 to $4 \mathrm{~kg}$ of weight in adjustable intervals, representing a step forward in size/weight terms.

The tray has three rotation points so that the dentist can place it in the right position with one hand and adapt it to their preferred way of working.

It is balanced by sets of elastic rings to give the tray better, more secure balance than conventional springs. It also features a removable panel to carry two standard instrument trays.

Thanks to its exclusive design, the tray can be adjusted according to the weight it has to bear at any time $(0-1 \mathrm{~kg}, 1-2 \mathrm{~kg}, 2-4 \mathrm{~kg})$ to ensure smooth movement. It also includes a manual brake that allows movement to be locked.

It is made from stainless steel to ensure a

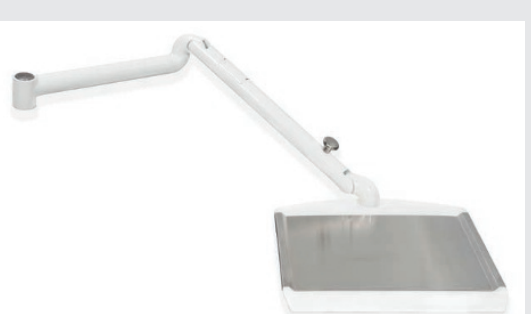

lasting, efficient product. The ergonomic design offers better handling and makes it easy to clean and disinfect.

More information is available at www. ancar-online.com. 